\title{
LUMBAR INTERVERTEBRAL DISC ALLOGRAFT TRANSPLANTATION: HEALING AND REMODELLING OF THE BONY STRUCTURE
}

\author{
Y-C. Huang ${ }^{1,2, \S}$, J. Xiao $^{3, \S}$, V.Y.L. Leung ${ }^{1}$, W.W. Lu ${ }^{1}, Y . \mathrm{Hu}^{1}$ and K.D.K. Luk ${ }^{1, *}$
}

${ }^{1}$ Department of Orthopaedics and Traumatology, The University of Hong Kong, Hong Kong SAR, China ${ }^{2}$ Shenzhen Engineering Laboratory of Orthopaedic Regenerative Technologies, Orthopaedic Research Center, Peking University Shenzhen Hospital, Shenzhen, China

${ }^{3}$ Department of Joint Surgery, Nanfang Hospital, Southern Medical University, Guangzhou, China

$\S$ Y-C. Huang and J. Xiao contributed equally to this work.

\begin{abstract}
Previous human study suggested that fresh-frozen intervertebral disc allograft transplantation can relieve neurological symptoms and restore segmental kinematics. Before wide clinical application, research into the pathophysiology of the postoperative disc allograft is needed. One important question that remains to be answered in disc allografting is the healing process of the host-graft interface and the subsequent change of the endplates. With the goat model for lumbar disc allografting, histology, micro-computed tomography analysis, scanning electron microscopy and energy-dispersive X-ray spectroscopy mapping were applied to evaluate the healing of the host-graft interfaces, the remodelling of subchondral bone, and the changes of the bony and cartilaginous endplates after transplantation. It was found that healing of the host-graft interfaces started at 1.5 months and was completed at 6 months by natural remodelling. This bony remodelling was also noted in the subchondral bone area after 6 months. The bony endplate was well preserved initially, but was gradually replaced by trabecular bone afterwards; on the other hand, the cartilaginous endplate became atrophic at 6 months and nearly disappeared at the final follow-up. Collectively, after intervertebral disc allograft transplantation, bony healing and remodelling were seen which ensured the stability and mobility of the disc-transplanted segment, but the integrity of bony and cartilaginous endplates was gradually lost and nearly disappeared finally.
\end{abstract}

Keywords: Intervertebral disc, allograft, transplantation, bony healing, remodelling, endplate.

\author{
*Address for correspondence: \\ Prof. Keith D.K. Luk \\ Department of Orthopaedics and Traumatology \\ The University of Hong Kong \\ 5/F Professor Block, Queen Mary Hospital \\ Pokfulam, Hong Kong SAR \\ China
}

Telephone Number: +85222554254

FAX Number: +852 28174392

E-mail: hrmoldk@hku.hk

\section{Introduction}

Intervertebral disc (IVD) degeneration has been widely recognised as the major generator of neck and back pain. It was found that about $20 \%$ of people in their teens had mild IVD degeneration (Boos et al., 2002), and that IVD degeneration increases significantly with age (Miller et al., 1988). When the secondary sequels, such as neck and back pain, are produced, treatment is required. Currently, the only medical interventions available are surgical measures to remove possible pain sources and to restore IVD biomechanical function (Huang et al., 2013). However, there has been a surge of interest in developing alternative methods of treatment focussed on biological methods of repairing or regenerating the IVD (Huang et al., 2014; Sakai et al., 2015).

Recently, an innovative treatment for severe IVD degeneration, using fresh-frozen IVD allograft transplantation was successfully developed in primates (Luk et al., 2008), and was finally used in 13 patients, where the allografts have provided acceptable clinical outcomes for up to 10 years (Ruan et al., 2007; Ding et al., 2016). Here, the neurological symptoms, and the motion and stability of the spinal unit improved significantly, although degeneration of the transplanted IVD allograft was observed in some cases at long-term follow-up (Ruan et al., 2007). In patients who are asymptomatic after IVD allografting, it is not ethical to perform a biopsy, as this could accelerate the degeneration of the allograft. Thus, the natural history of the postoperative IVD allograft and its degenerative mechanisms are still unclear.

Structurally, the IVD allograft usually carries 2-3 mm of vertebral bone at both the cranial and caudal ends in order to protect its integrity (Ruan et al., 2007; Luk et al., 2008; Lam et al., 2012); a bony gap hence exists between the allograft and the host recipient bone. Insufficient healing and remodelling of the host-graft interfaces may affect the success of IVD allografting as well as the stability and mobility of the IVD-transplanted segment. Hence, the first aim in this study was to investigate whether and how the IVD allograft was healed with the host vertebral bone using the newly-developed goat model for lumbar IVD allografting (Xiao et al., 2015).

Bony and cartilaginous endplates (BEP and CEP) have been demonstrated to play an important role in the maintenance of IVD health by providing mechanical support to the nucleus and annulus, preventing the leakage of macromolecules, and facilitating the transport of nutrient/metabolite into and out of the IVD (Moore, 

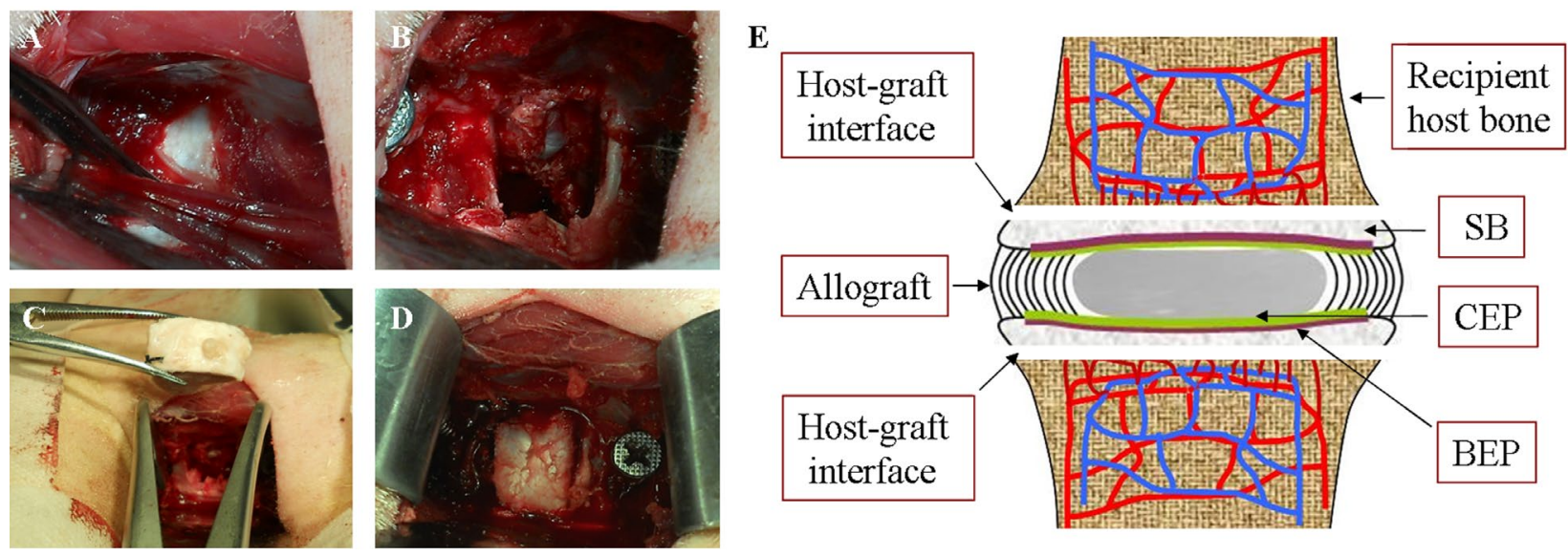

Fig.1. The surgery technique for lumbar fresh-frozen IVD allograft transplantation and the schematic anatomy of lumbar spine after IVD allografting. (A-D) The "retro-psoas" approach was used for lumbar IVD exposure in goats and the recipient slot was prepared at L4/L5; the IVD allograft with the most compatible size was selected, thawed and implanted without internal fixation. The two temporary screws were removed and the goats were allowed free mobilisation immediately after recovering from the anaesthesia. (E) In this technique, in order to protect the integrity of disc, 2-3 mm vertebral bone and the subchondral bone were transplanted. Host-graft interfaces hence existed between the IVD allograft and the recipient host bone until complete bony healing. Thus, healing and remodelling of the host-graft interface was essential for the stability of the allograft-transplanted segment, and the change of the bony and cartilaginous endplates may play a critical role in the reestablishment of nutrient channels in IVD allografts. Abbreviations: SB, subchondral bone; CEP, cartilaginous endplate; BEP, bony endplate.

2006; Lotz et al., 2013). Immediately after surgery, the IVD allograft existed in an ischaemic environment until reestablishment of the nutrient pathway during bone healing (Luk et al., 2008; Huang et al., 2014). Understanding the change in the endplates (EPs) of the postoperative IVD allograft is probably able to uncover the re-establishment of nutrient channels. The second aim in this study is thus to investigate the structural change of the EPs in the IVD allograft after transplantation.

Therefore, in the present study, using the newlydeveloped surgical techniques (Xiao et al., 2015), we performed IVD allografting in 15 goats (Fig. 1A-D); then we investigated how the IVD allograft was healed with host bone and how the EPs were changed after transplantation. Anatomically, the following steps (Fig. 1E) were included: 1) healing of the host-graft interfaces, 2) remodelling of the subchondral bone, and 3) changes of the EPs.

\section{Materials and Methods}

\section{Animals}

The research proposal has been approved by the Committee on the Use of Live Animals in Teaching and Research, the University of Hong Kong (CULATR1872-09). Totally, 20 male goats between 6 and 9 months and weighing between 20 and $27.5 \mathrm{~kg}$ were used in this study. These goats are sexually mature as early as 6 months, but they still have growth capacity as the growth plates are still present. Out of these 20 goats, 5 goats were used as IVD allograft donors, and the remaining 15 goats as allograft recipients.

\section{Fresh-frozen IVD allograft transplantation in goat lumbar spine}

Five lumbar discs from L1/L2 to L5/L6 levels were selected as allografts, hence 25 IVD allografts were harvested from the 5 donor goats. The allografts were cut with growth plate and they were then immersed in the cryopreservative solution; the temperature was decreased gradually to $-80{ }^{\circ} \mathrm{C}$ and finally the allografts were preserved in liquid nitrogen for less than 1 month until transplantation. During preparation of the recipient slot, the nucleus pulposus, posterior annular fibrosus, the endplates and the growth plates were removed. Before insertion, the IVD allograft of the most compatible size was selected and thawed completely at $37^{\circ} \mathrm{C}$. The mean lateral width, anteroposterior width and height of the IVD allografts after trimming were $20.2 \mathrm{~mm}, 13.4 \mathrm{~mm}$ and $10.0 \mathrm{~mm}$, respectively. Then, IVD allografting without internal fixation was performed at lumbar L4-L5 (Fig. 1A-D) using the previously developed surgical techniques (Xiao et al., 2015). The goats were allowed free mobilisation immediately after recovering from the general anaesthesia.

\section{Animal sacrifice and specimen harvest}

Groups of 5 goats were sacrificed at 1.5, 6 and 12 months postoperatively $(n=5)$. The disc-transplanted segment, along with the adjacent levels, were then harvested en bloc and fixed in $4 \%$ paraformaldehyde in phosphate-buffered saline for $7 \mathrm{~d}$.

\section{Micro-computed tomography (CT) scanning and analysis}

The 15 spine specimens were scanned using the SkyScan 1076 micro-CT system (SkyScan, Kontich, Belgium) to 

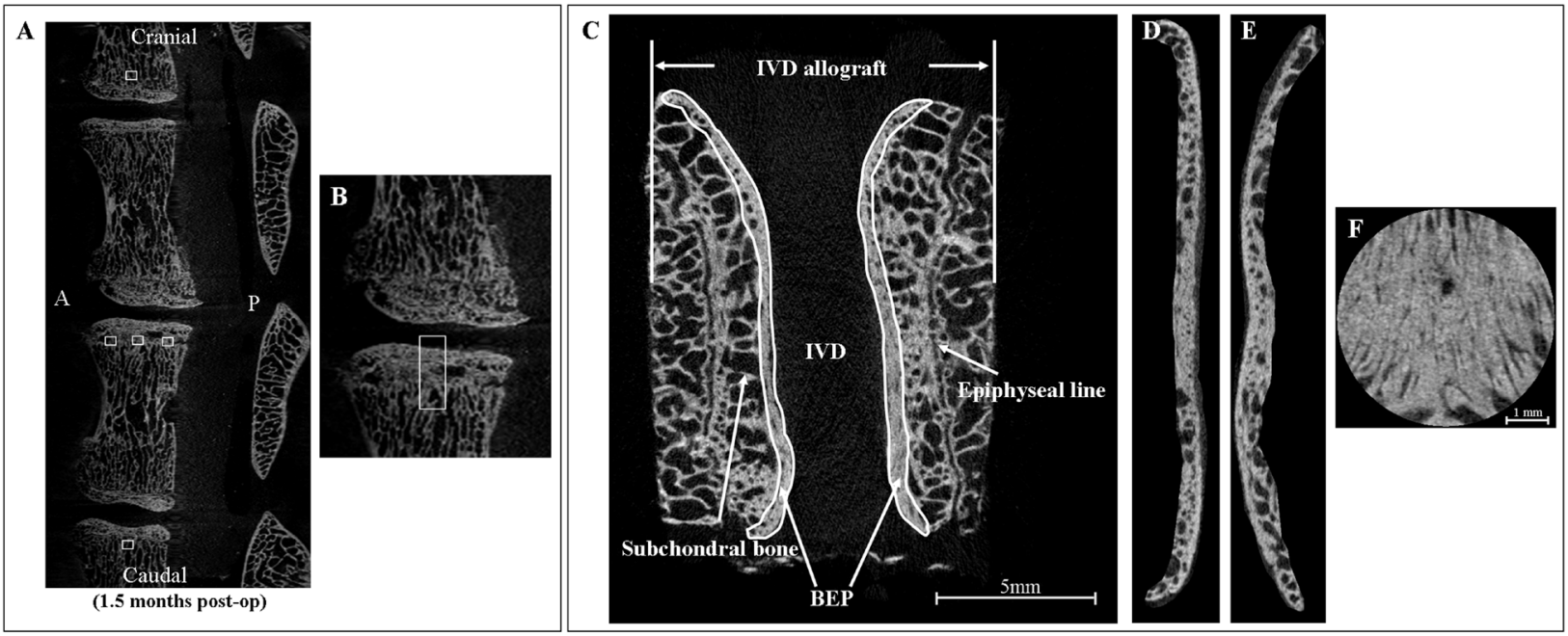

Fig.2. Micro-CT analysis for the bony structure at the host-graft interfaces and that of the bony endplate. (A) Three ROIs with the size of $1.73 \times 1.57 \times 1.73 \mathrm{~mm}^{3}$ (indicated by the white rectangle) were selected at the anterior, centre and posterior of the host-graft healing sites based on the CT images of the lumbar spine after IVD allografting; two ROIs with the same size (indicated by the white rectangle) were used at the adjacent levels as controls. (B) A cylinder with a diameter of $1.734 \mathrm{~mm}$ and a length of $5.184 \mathrm{~mm}$ was extracted at the centre of the vertebral body crossing the IVD allograft for 3D reconstruction to grossly observe the healing process of the host-graft interface after transplantation. (C) CT image illustrating the bony structure of IVD allograft before transplantation. Scale bar $=5 \mathrm{~mm}$. (D-E) Both the cranial and caudal bony EPs were identified on the CT images and then extracted as ROIs for $3 \mathrm{D}$ reconstruction. (F) A cylinder with a mean diameter of $4.49 \mathrm{~mm}$ and mean length of $0.344 \mathrm{~mm}$ was placed at the centre of the bony EP to see the channels in the bony EP after IVD allografting. Scale bar $=1 \mathrm{~mm}$. Abbreviations: A, anterior; P, posterior; BEP, bony endplate.

investigate the healing of host-graft interfaces and the change of bony EP after IVD allografting. Five freshfrozen IVD allografts without implantation were scanned as control for bony EP evaluation. After micro-CT analysis, the images were acquired at a scan resolution of $17.3 \mu \mathrm{m}$, a voltage of $60 \mathrm{kV}$, a current of $167 \mathrm{~mA}$ and an exposure time of $4.4 \mathrm{~s}$. Three-dimensional reconstructions were generated using NRecon (SkyScan, Kontich, Belgium). A total number of 2400 to 3200 coronal slices of microCT images were acquired from each specimen. The total scanning time for each specimen was approximately $6.0 \mathrm{~h}$. The raw data were reconstructed as Tag Image File Format images.

To investigate the healing of host-graft interfaces, three regions of interests (ROIs) with a size of $1.73 \times 1.57 \times 1.73 \mathrm{~mm}^{3}$ were placed at the anterior, centre and posterior of the host-graft interfaces; two ROIs of the same size were selected at the centre of the adjacent vertebral bodies near the epiphyseal line (Fig. $2 \mathbf{A})$. The mean of the values at the three positions of the host-graft interfaces was calculated and compared with that of two adjacent vertebral ROIs as normal controls to evaluate bone formation and architecture according to the following parameters: bone volume over total volume (BV/TV, \%), trabecular thickness (Tb.Th, mm), trabecular number (Tb.N, 1/mm), trabecular separation (Tb.Sp, mm), trabecular pattern factor (Tb.Pf, 1/mm), structure model index (SMI), degree of anisotropy (DA) and connectivity density (Conn.Dn, $1 / \mathrm{mm}^{3}$ ). A cylinder with a diameter of
$1.734 \mathrm{~mm}$ and a length of $5.184 \mathrm{~mm}$ was extracted at the centre of the vertebral body crossing the disc allograft for $3 \mathrm{D}$ reconstruction and gross observation of the healing at host-graft interfaces after transplantation (Fig. 2B).

Furthermore, the bony EPs at both the cranial and caudal ends were identified, and all of the contours (ROI) were drawn on the CT images using a semi-automated contouring approach (Fig. 2C-E) with the CT Analyser software (SkyScan, Kontich, Belgium). The extracted EP tissues were segmented using a global threshold for 3D analysis and 3D reconstruction; from the $3 \mathrm{D}$ reconstructed data, a cylinder with a mean diameter of $4.49 \mathrm{~mm}$ and mean length of $0.344 \mathrm{~mm}$ was used at the centre of the bony EP to observe the marrow contact canals (Fig. 2F). During analysis, the cranial and caudal EPs were analysed together to observe the continuous changes at 1.5, 6 and 12 months after disc transplantation. According to the literature (Rutges et al., 2011; Wang et al., 2011; Fields et al., 2012), the following micro-structural parameters were calculated to evaluate the bony EP structure: BV/TV $(\%)$, thickness $(\mathrm{mm})$, Conn.Dn $\left(1 / \mathrm{mm}^{3}\right)$ and total porosity (Po (tot), \%). The thickness of the bony EP was defined as the distance between the cranial and caudal surfaces; this varies at different sites within the bony EP, so only the mean thickness was measured. Total porosity is the volume of all open plus closed pores as a percent of the total bony EP volume. 
Scanning electron microscope (SEM) analysis and energy-dispersive X-ray spectroscopy (EDX) mapping

After micro-CT scanning, the transplanted allograft was separated and cut mid-sagittally using a band saw (EXAKT 300CP Band System, Norderstedt, Germany). Para-midsagittal tissue slices (2-3 mm thick) were then cut from the half of IVD allograft specimen without decalcification; these slices were air dried and gold sputtered for SEM and EDX analysis (Hitachi S-3400N Variable Pressure SEM, Hitachi, Tokyo, Japan) for morphological observation and assessment of the distribution profile of Calcium $(\mathrm{Ca})$ and Phosphorus (P) at the bony structure of the IVD allograft. Ca was noted by green dots while $\mathrm{P}$ was noted by red dots in the SEM images.

\section{Histological staining}

The other half of the transplanted IVD allografts and the five fresh-frozen IVD allografts without implantation were decalcified, dehydrated, embedded in paraffin wax and finally cut into $5 \mu \mathrm{m}$-thick sections for haematoxylin and eosin (HE), Masson-trichrome (MT) and Safranin O/fast green (SF) staining. Picro-sirius red (PSR) staining was conducted to observe the collagen profile using a polarised light microscope (Leica DM 4000B, Leica, Mannheim, Germany) following previous literature (Junqueira et al., 1982).

\section{Statistical analysis}

All values were expressed as mean \pm standard deviation (SD). Two-way ANOVA (two groups (host-graft interfaces and adjacent controls $) \times 3$ time points $(1.5$ months post-op, 6 months post-op and 12 months post-op)) was performed to detect the effect of treatment and time on the changes in bone morphological parameters (BV/TV, Tb.Th, Tb.N, Tb.Sp, Tb.Pf, SMI, DA and Conn.Dn) as measured by the micro-CT scanning; then, post-hoc comparisons using the Least Significant Difference (LSD) model were used to determine the difference between the groups at different time slots. One-way ANOVA with a LSD post-hoc analysis was conducted to compare the microstructural parameters (BV/TV, thickness, Conn.Dn and Po (tot)) of the bony EP at the different time slots after transplantation. Statistical analysis was performed with SPSS 16.0 software (SPSS Inc., Chicago, IL, USA), and significance was accepted at $p<0.05$.

\section{Results}

\section{Progressive healing of the host-graft interfaces}

All of the IVD allografts were well seated and remained so throughout the follow-up period without subluxation or dislocation (Huang et al., 2016). After micro-CT analysis, the bony interface was noted at 1.5 months, but it became invisible after 6 months; and finally the architecture of healing sites was similar with that of the normal adjacent control with minor differences (Fig. 3A).

The dependent variables, the bone morphological parameters, were normally distributed and there was homogeneity of variance between groups as assessed by Levene's test. These data were analysed by two-way ANOVA to detect difference due to group and time. The group differently affected the bone morphological parameters: $\mathrm{BV} / \mathrm{TV}(p=0.000)$, Conn.Dn $(p=0.000)$, Tb.N $(p=0.000)$, Tb.Th $(p=0.006)$, Tb.Pf $(p=0.001)$, $\operatorname{SMI}(p=0.004)$ and DA $(p=0.000)$, while the time affected Tb.Th $(p=0.008), \operatorname{Tb} . P f(p=0.022)$ and SMI $(p=0.047)$; but the interaction of group $\times$ time showed that only DA was significantly regulated $(p=0.029)$. To understand the healing process of host-graft interface over time, Fig. 3B showed that at 1.5 months the host-graft interfaces had more new bone formation with a plate-like and wellconnected architecture, because the interfaces had higher BV/TV $(p=0.002)$, Tb.Th $(p=0.014)$, Tb.N $(p=0.000)$ and Conn.Dn $(p=0.000)$, and lower Tb.Pf $(p=0.001)$ and $\operatorname{SMI}(p=0.002)$ compared to the mean of adjacent controls. The bony remodelling was undergoing after 6 months as the means of Tb.N $(p=0.037)$ and Conn.Dn $(p=0.042)$ were greater than those of the adjacent control, and it remained at the last follow-up with higher Tb.N $(p=0.031)$ and Conn.Dn $(p=0.014)$, and lower DA $(p=0.000)$.

Resorption of the vertebral bones and extensive immature bone formation were noted at the host-graft interfaces after 1.5 months; thus, a bony bridge connecting the allograft with the host bone was formed (Fig. 4A-D). At this stage, the healing was not yet complete because granulation tissues without the presence of $\mathrm{Ca}$ and $\mathrm{P}$ were also present (white arrow in Fig. 4D). At 6 months, dense and mature trabecular bone was identified (Fig. 4E-H); complete healing was evident and bone marrow cavities had reformed. At 12 months, typical trabecular bone was present at the host-graft interface after continuous bony remodelling (Fig. 4I-L).

\section{Remodelling of the subchondral bone}

After the fresh-frozen process, the osteocytes residing inside the lacuna could survive in the subchondral bone, but aggregation of the bone marrow stroma and even destruction of the bone marrow architecture were evident (Fig. 5A-C). After 1.5 months, some empty lacunae were found and the bone marrow cavity was full of granulation tissues (Fig. 5D-F). In the form of creeping substitution, the subchondral bone was basically replaced by the new bone, with many viable osteocytes lying in the lacunae (Fig. 5G-I); additionally, typical bone marrow was formed finally (Fig. 5J-L).

\section{Disappearance of bony endplate}

Goat bony EP is a thin layer of cortical bone with a nearly oval shape and it is penetrated by many marrow contact channels with a diameter less than $250 \mu \mathrm{m}$ (Fig. 6A); it was preserved at 1.5 months; however, the structural integrity at 6 months was found to be destroyed and finally the bony EP was basically replaced by trabecular bone. As shown in Fig. $6 \mathbf{B}$, the mean BV/TV of bony EP decreased from $83.39 \%$ to $68.72 \%$ after 6 months $(p=0.000)$, although no significant reduction was found at 1.5 months. The bony EP thickness was decreased from $0.41 \pm 0.06 \mathrm{~mm}$ to 0.25 $\pm 0.03 \mathrm{~mm}$ at 1.5 months $(p=0.000)$ and was maintained at this level afterwards. The connectivity density in the bony EP increased remarkably, from $47.58 \pm 17.42$ before 


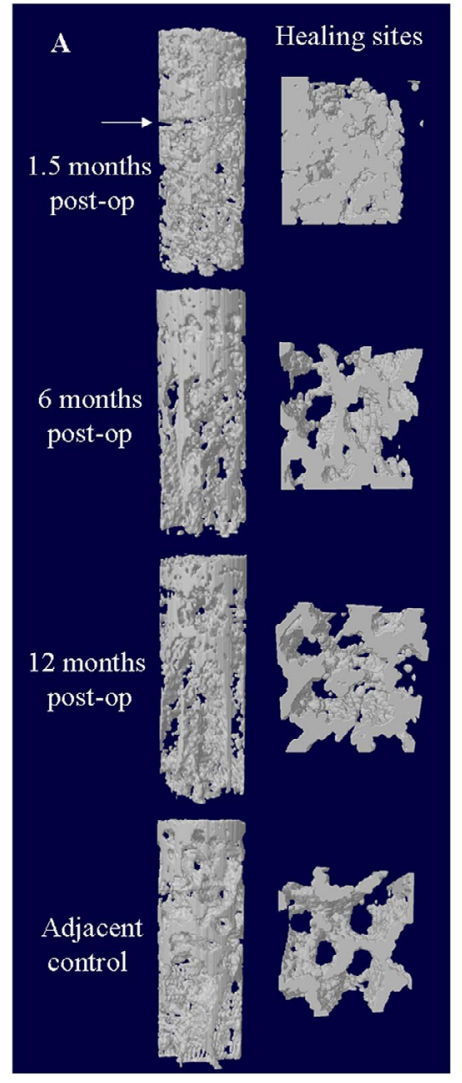

\begin{tabular}{|c|c|c|c|c|}
\hline & Groups & $\begin{array}{c}1.5 \text { months } \\
\text { post-op }\end{array}$ & $\begin{array}{l}6 \text { months } \\
\text { post-op }\end{array}$ & $\begin{array}{l}12 \text { months } \\
\text { post-op }\end{array}$ \\
\hline \multirow{2}{*}{ BV/TV $(\%)$} & Host-graft interfaces & $39.84 \pm 9.26 * *$ & $29.18 \pm 8.85$ & $35.87 \pm 8.94$ \\
\hline & Adjacent controls & $22.81 \pm 7.68$ & $20.65 \pm 7.15$ & $26.29 \pm 4.20$ \\
\hline \multirow{2}{*}{ Tb.Th (mm) } & Host-graft interfaces & $0.17 \pm 0.01 \%$ & $0.15 \pm 0.01$ & $0.16 \pm 0.02$ \\
\hline & Adjacent controls & $0.14 \pm 0.01$ & $0.13 \pm 0.01$ & $0.16 \pm 0.01$ \\
\hline \multirow{2}{*}{ Tb.N (1/mm) } & Host-graft interfaces & $2.50 \pm 0.25^{* *}$ & $1.97 \pm 0.35^{*}$ & $2.15 \pm 0.29 *$ \\
\hline & Adjacent controls & $1.57 \pm 0.40$ & $1.51 \pm 0.41$ & $1.67 \pm 0.24$ \\
\hline \multirow{2}{*}{ Tb.Sp (mm) } & Host-graft interfaces & $0.34 \pm 0.10$ & $0.35 \pm 0.07$ & $0.33 \pm 0.07$ \\
\hline & Adjacent controls & $0.40 \pm 0.05$ & $0.39+0.08$ & $0.39 \pm 0.04$ \\
\hline \multirow[b]{2}{*}{ Tb.Pf $(1 / \mathbf{m m})$} & Host-graft interfaces & $-2.36 \pm 4.88^{* *}$ & $4.38 \pm 2.83$ & $1.20 \pm 2.60$ \\
\hline & Adjacent controls & $6.09 \pm 4.47$ & $8.07 \pm 3.32$ & $4.16 \pm 1.53$ \\
\hline \multirow{2}{*}{ SMI } & Host-graft interfaces & $0.79 \pm 0.77 * *$ & $1.67 \pm 0.40$ & $1.30 \pm 0.42$ \\
\hline & Adjacent controls & $1.87 \pm 0.51$ & $2.07 \pm 0.39$ & $1.55 \pm 0.31$ \\
\hline \multirow{2}{*}{$\begin{array}{c}\text { Comn.Dn } \\
\left(1 / \mathbf{m m}^{3}\right)\end{array}$} & Host-graft interfaces & $47.70 \pm 15.56^{* *}$ & $32.05 \pm 12.67^{*}$ & $34.14 \pm 4.93^{*}$ \\
\hline & Adjacent controls & $2121+886$ & $18.60 \pm 7.17$ & $17.54 \pm 5.51$ \\
\hline \multirow{2}{*}{ DA } & Host-graft interfaces & $1.76 \pm 0.27 *$ & $2.10 \pm 0.37$ & $1.86 \pm 0.18^{* *}$ \\
\hline & Adjacent controls & $2.37 \pm 0.38$ & $2.22 \pm 0.29$ & $2.89 \pm 0.54$ \\
\hline
\end{tabular}

Fig.3. Healing and remodelling of host-graft interfaces by micro-CT analysis after IVD allografting. (A) Bony healing was found, but not completed yet; the host-graft interface (indicated by the white arrow) still existed after 1.5 months; complete healing was seen after 6 months. Finally, the bony structure at the healing sites became similar to that of adjacent controls after continuous remodelling. (B) Quantitative micro-CT analysis of the bony architectures at the host-graft interfaces and at the adjacent controls at 1.5, 6 and 12 months after IVD allografting. Two-way ANOVA analysis was done; ${ }^{*} p<0.05$ host-graft interfaces $v s$. adjacent controls, ${ }^{* *} p<0.01$ host-graft interfaces $v s$. adjacent controls.

implantation to $95.05 \pm 27.69$ at 1.5 months $(p=0.013)$ and to $133.63 \pm 60.63(p=0.000)$ at 6 months; additionally, a mean total porosity of $31.27 \%$ was found at 6 months, which was much higher in comparison to preoperative levels $(p=0.001)$. At the final follow-up, most of the bony EP had disappeared, so that the structural parameters of the bony EP were no longer measured. Histological staining and SEM-EDX mapping results further indicated the disappearance of the bony EP (Fig. 7A-I). The integrity of the bony EP was maintained at 1.5 months, but not at 6 months. Defects of the bony EP were documented, accompanied by extensive granulation tissue formation. At 12 months, new bone formation and bone loss were seen at the EP area and no typical EP-like structure remained; also bone marrow cavities were formed which opened directly to the disc tissue (Fig.7G-I).

\section{Atrophy of the cartilaginous endplate}

The chondrocytes could survive with normal cellular morphology and the cartilage matrix was also well maintained at the 1.5-month follow-up (Fig. 7J-K). At 6 months, disruption of the cartilaginous EP can be seen and most of the cartilage lacunae were empty, indicating that a large proportion of the resident chondrocytes were necrotic
(Fig. 7M-N). Only very few chondrocytes resident near the bone marrow cavity maintained the normal morphology. At the final stage, the cartilage matrix was remarkably atrophic and basically disappeared after replacement by the newly-formed matrix (Fig. 7P-Q).

The colours of collagen in the bony EP and cartilaginous EP were quite different, where the former was green and the latter was red which could be observed after PSR staining. These differences could be found after 1.5 months (Fig. $7 \mathbf{L}$ ); but followed by the disappearance of the bony EP and cartilaginous EP, the collagen in the original cartilaginous EP area (Fig. $7 \mathbf{O}$ and $\mathbf{R}$ ) was mixed after replacement by those with a yellow or green colour, which suggested that the fibre diameter decreased. Thus, the obvious and regular interface between the bony EP and cartilaginous EP was not preserved any more.

\section{Discussion}

As a natural alternative to bone autograft, bone allograft has long been widely used for filling bone voids and augmenting facture healing (Bauer et al., 2000). The bone allograft undergoes a repair and remodelling 
1.5 months post-op
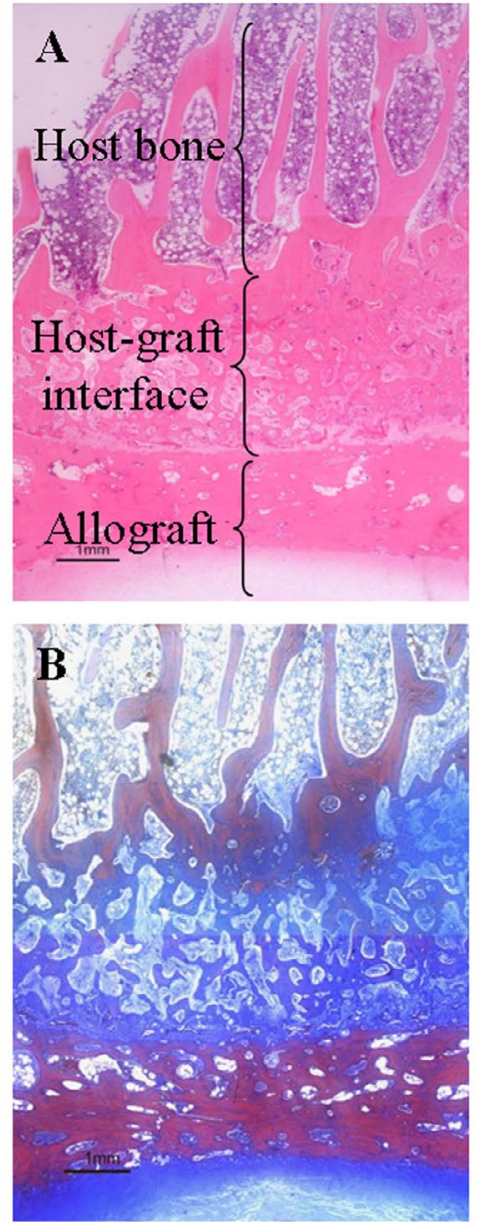

MT

SEM
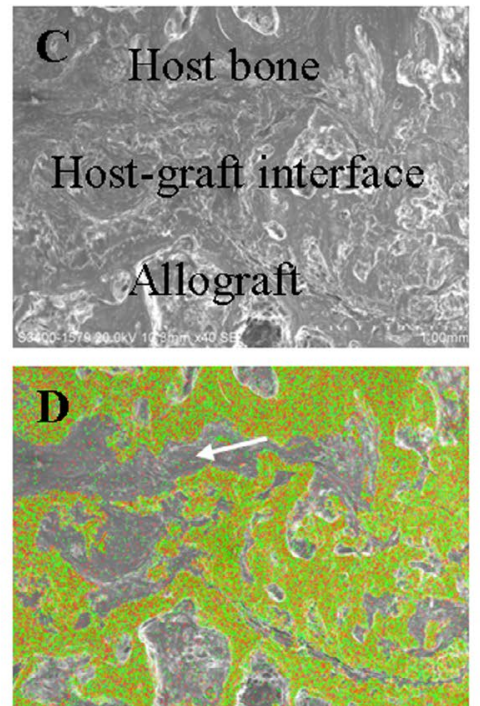

6 months post-op
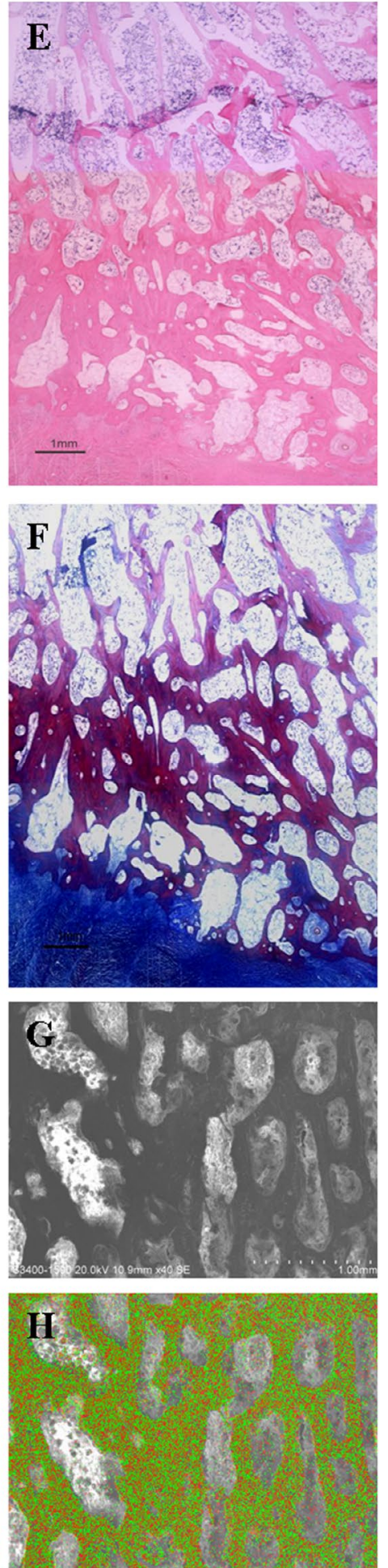

12 months post-op
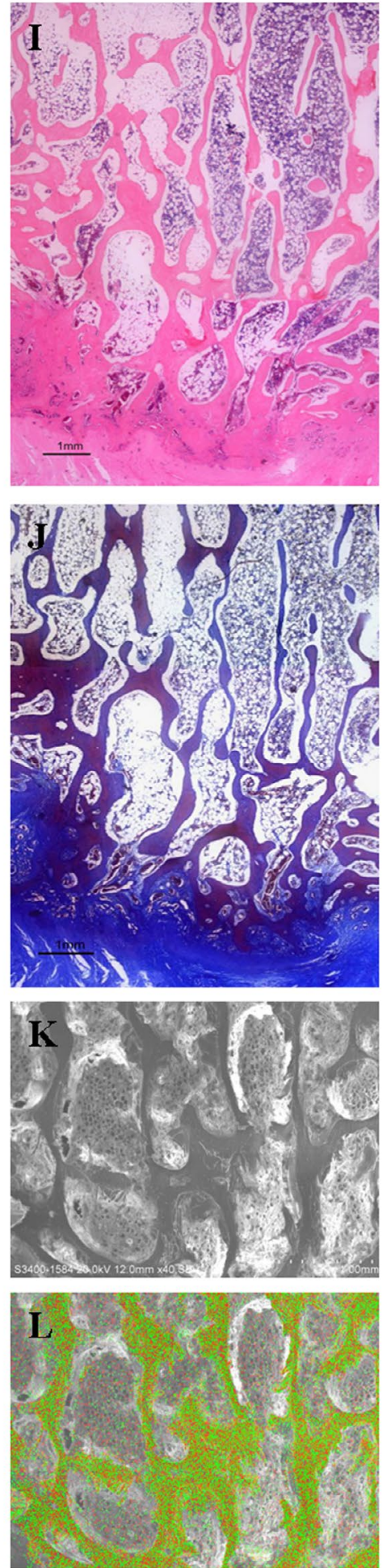

Fig.4. Histology and SEM-EDX mapping illustrated the healing and remodelling of the host-graft interfaces after 12 months. (A-D) At the 1.5-month follow-up, a large amount of immature woven bone formation (indicated by the brackets) was found, but the healing was not completed yet, as the granulation tissue without distribution of $\mathrm{Ca}$ and $\mathrm{P}$ (indicated by the white arrow) was presented in the healing sites. (E-H) Complete healing was found and the host-graft interfaces disappeared after 6 months. (I-L) At final follow-up, the newly-formed bone architecture was similar to the normal one. Scale bar for all the histology and SEM figures is $1 \mathrm{~mm}$. Abbreviation: HE, haematoxylin and eosin; MT, Masson-trichrome; SEM, scanning electron microscope; EDX, energy-dispersive X-ray spectroscopy; $\mathrm{Ca}$, calcium; P, phosphorus. 


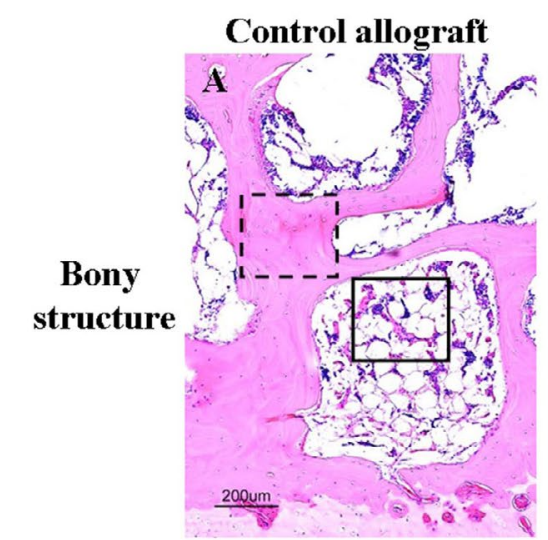

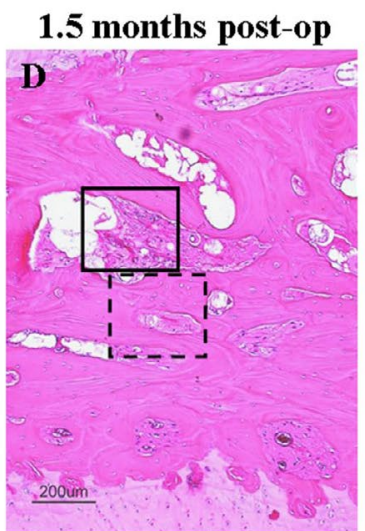

6 months post-op
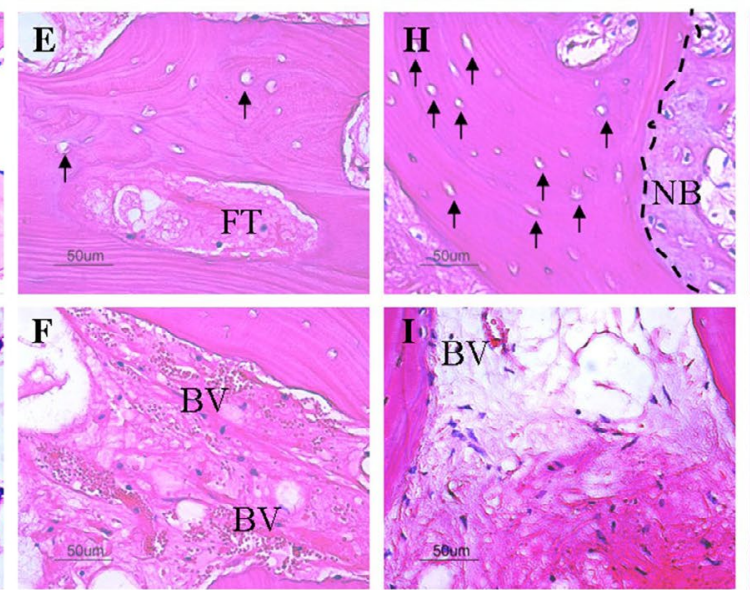

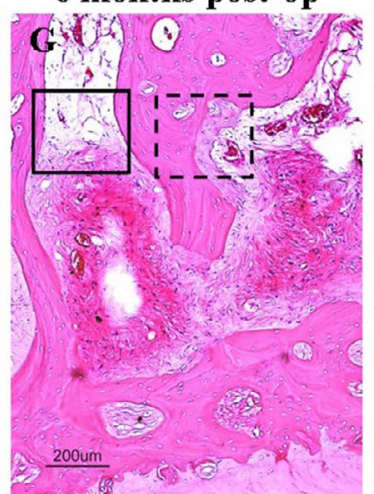

12 months post-op
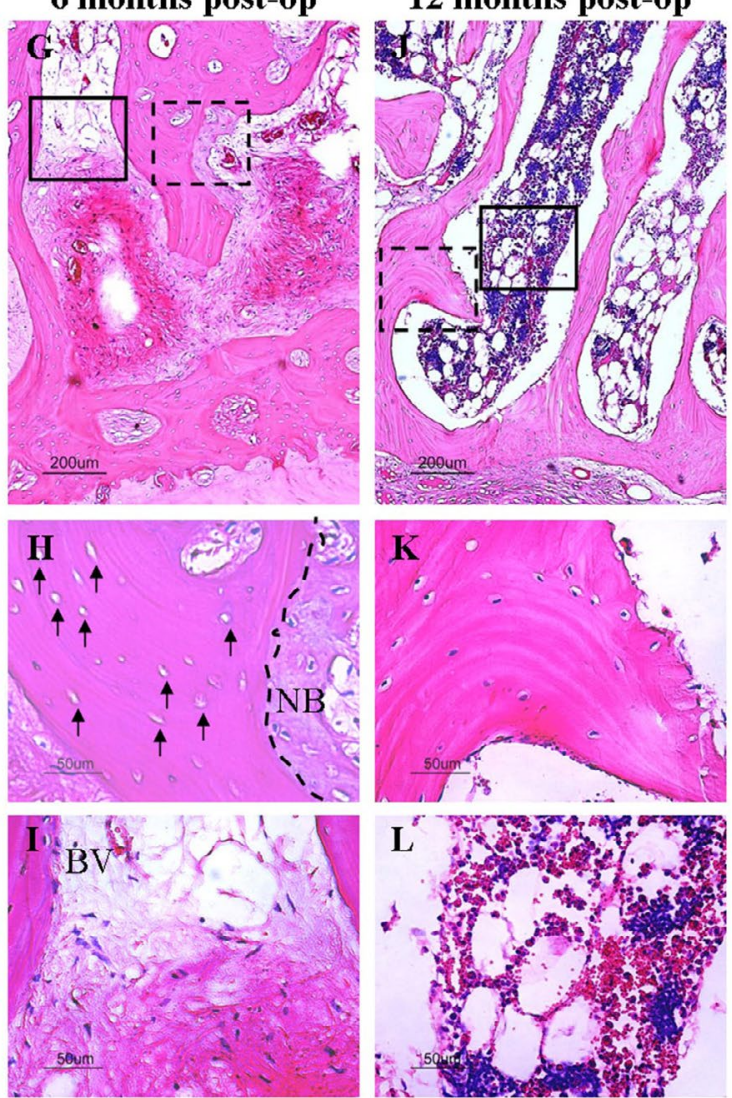
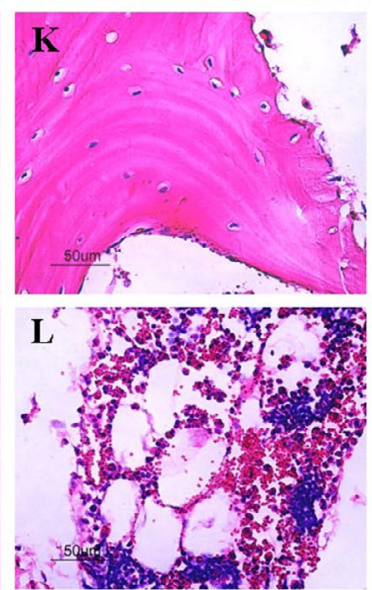

Fig.5. HE staining results indicated the remodelling process of the subchondral bone and reformation of the bone marrow at 12 months after IVD transplantation. After HE staining, figures with higher magnification of the areas at the dotted boxes and at the full-line boxes were captured to illustrate the morphology of trabecular bone and the bone marrow cavity, respectively. (A-C) In the fresh-frozen IVD allografts without implantation (Control allograft), the osteocytes residing inside the lacuna of trabecular bone survived well, but aggregation of the bone marrow stroma and even destruction of the bone marrow architecture were evident. (D-F) At 1.5 months postoperatively, granulation tissue was observed at the bone marrow cavity and some empty lacunae were seen. (G-I) After 6 month, extensive empty osteocyte lacunae in the trabeculae and new bone formation were found. (J-L) At the final follow-up, the subchondral bone was replaced by trabecular bone and bone marrow was reformed. The black arrows point out the empty osteocyte lacunae in the trabeculae. Abbreviations: FT, fibrotic tissue; NB, new bone; BV, blood vessels.

process to initiate a healing response from the host bed that will produce new bone at the host-graft interfaces and afterwards within the porous allograft (Delloye et al., 2007; Di Bella et al., 2010; Messora et al., 2013). Bony healing and remodelling were observed in the bony structure of the postoperative IVD allograft, although the amount of bone transplanted during IVD allografting was very small. At the host-graft interfaces, although most of the CT parameters and the morphology of newly-formed trabecular bone were similar to that of adjacent controls, the Tb.N, Conn.Dn and DA were different. At the bony interface after IVD allografting for 6 months, a higher amount of trabecular bone with more connections was evident which may enhance the stiffness (Odgaard et al., 1993). Meanwhile, the DA is one of the most important determinants of mechanical strength (Kim et al., 2013); a lower DA means that the structure of trabecular bone at the healing sites was more anisotropic than the normal adjacent controls, suggesting more resistance to external force. This bony healing and remodelling at the host-graft interface is essential to restore the stability and mobility of the lumbar spine after IVD allografting.

As a structural shield, the EP prevents the bulging of IVD into the adjacent vertebral bodies (Roberts et al., 1996). As mechanical interfaces, the EP disperses the compressive loading experienced by the vertebra-disc complex (Ferguson et al., 2003). Importantly, the EP is the predominant regulator for nutrient transportation, as the nutrient supply to most of the disc cells has to pass through the EP canal network by diffusion (Urban et al., 2004; Yamaguchi et al., 2015). Both bony and cartilaginous EPs were preserved initially, but their integrity was gradually lost over time. Possible interpretations are as follows: First, this phenomenon is a normal bone remodelling process, similar to that found in the host-graft interfaces and the subchondral bone; the bony EP of the allograft was replaced by the host trabecular bone in the form of creep substitution as new bone formation and bone loss 

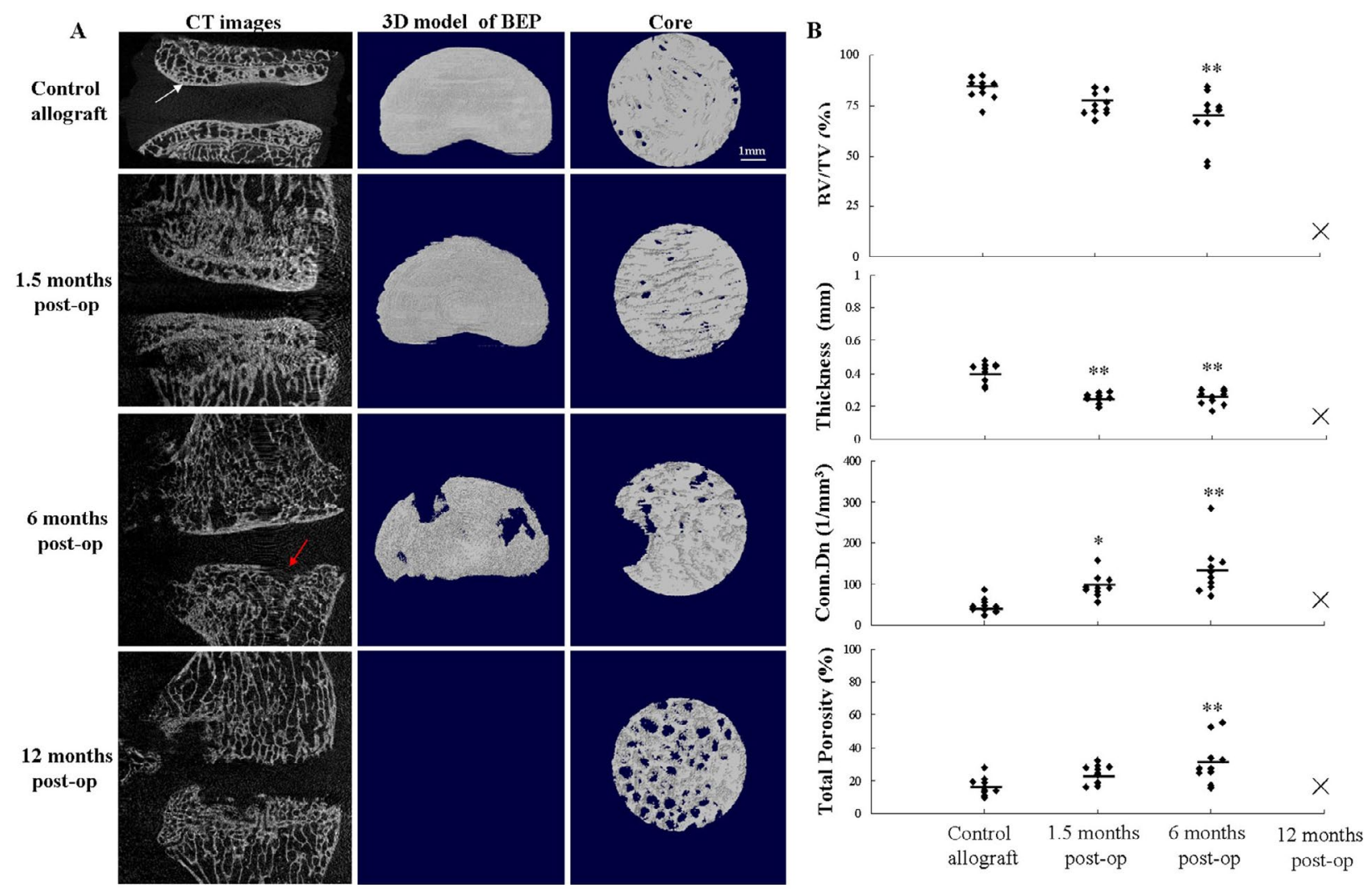

Fig.6. Micro-CT data determined the change of the bony EP after IVD allografting. (A) The bony EP (indicated by the white arrow) was a thin layer of cortical bone with a nearly oval shape and it was penetrated by many marrow contact channels. The bony EP was well preserved after 1.5 months, but it was gradually replaced by trabecular bone after continuous remodelling; obvious defects (indicated by the red arrow) were seen based on the CT images and 3D reconstructed model after 6 months. Finally, most of the bony EP was replaced by trabecular bone; direct contact of the IVD with the vertebral bone was generated, as the diameter of the bony EP canal was increased. (B) Quantitative micro-CT analysis of the bony EP at 1.5, 6 and 12 months after IVD allografting. Both the cranial and caudal bony EPs were included for quantitative analysis, thus the total number of bony EP in each time slot was 10. At 12 months postoperatively, the typical bony endplate structure could not be found, thus the analysis was not performed. ${ }^{*} p<0.05 v s$. preoperative value, ${ }^{* *} p<0.01 v s$. preoperative value. Abbreviation: BEP, bony endplate.

was seen. The second reason may have to do with microfractures. As the structural transition between the hard vertebral bone and the soft disc tissue, the EP is a region of high stress (Rodrigues et al., 2012), and it is the 'weak link' in the lumbar spine (Yoganandan et al., 1994; Adams et al., 2011). Alterations in compressive stress may result in micro-fractures in the bony EP during surgery or after implantation, thus leading to cell apoptosis and increasing the possibility of disc disruption and degeneration (Haschtmann et al., 2008; Dolan et al., 2013). One should see micro-callus if micro-fractures happened in normal bone; however, in this case the micro-fractures occurred in the allograft in which the vascularity has not yet been re-established fully. The absence of micro-callus therefore cannot exclude micro-fractures.

Beyond the mechanical and nutritional functions, the EP provides secure anchorage for the collagen network arising from the nucleus and annulus in order to realise the structural integration (Rodrigues et al., 2012; Wade et al., 2012; Wade et al., 2014). In the ovine disc, the EPnucleus integration was achieved by nodal fibrils to provide the nucleus with a form of tethered mobility (Wade et al., 2012; Wade et al., 2014); the EP-annulus was integrated by annular fibre bundles subdividing into sub-bundles to form a three-dimensional multi-leaf morphology (Rodrigues et al., 2012). These intrinsically nodal anchoring systems should be the results of the adaptive response to the biomechanical environment in the disc. Owing to the difference in the methods of sections preparation, the nodal attachment points were not easy to be observed in the junction of the bony and cartilaginous EP, but many aligned collagen fibres inserting in the bony EP were found after disc transplantation. These fibres may belong to the nodal anchoring systems, but they became loose and irregular in the EP region after defects were formed at 12-moth follow-up. Such changes are important because they may lead to an uneven distribution of the loading across the nucleus and annulus, and thus may contribute to the mild degeneration of disc allograft after transplantation.

The concept of IVD allograft transplantation has been developed for nearly 20 years; this is the first study to determine the bony healing and remodelling process of IVD 

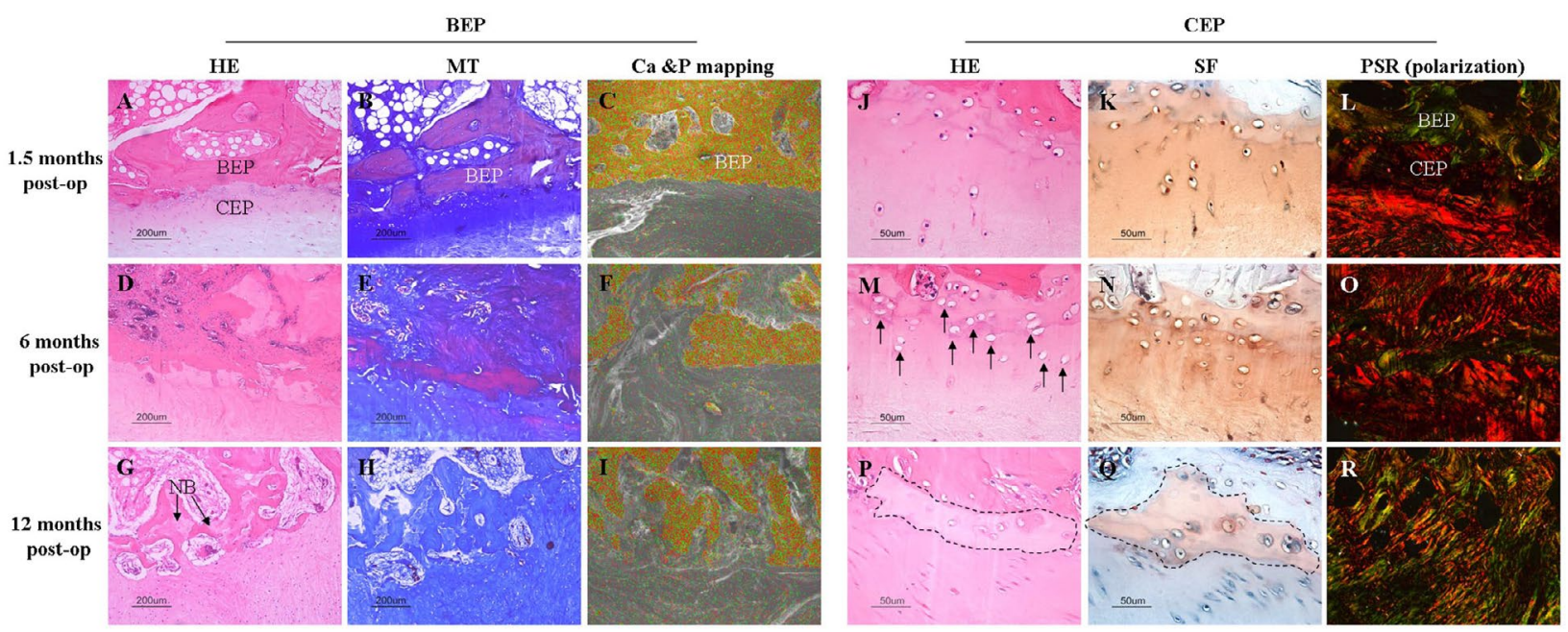

Fig.7. Morphological changes of the bony and cartilaginous EPs after IVD allografting. (A-C) The integrity of bony EP was well maintained at 1.5 months, and it was easy to be distinguished from the CEP. (D-F) After 6 months, defects at the bony EP were seen and the integrity was not preserved anymore. (G-I) At the final follow-up, the bony EP was replaced by newly-formed trabecular bone (NB, indicated by black arrows) and bone remodelling was clearly evident by HE and MT staining. (J-L) Similarly, the cartilaginous EP was well preserved at 1.5 months, and typical chondrocytes embedded in the Safranin O-positive matrix could be clearly identified. (M-O) Empty cartilage lacunae were notably determined (indicated by black arrows) in the Safranin O-positive matrix at 6 months postoperatively; the junction between the bony and cartilaginous EPs was not easily distinguishable. (P-R) After 12 months, typical chondrocytes resident in the cartilage lacuna were not found, and the cartilage matrix was remarkably atrophic (the area within dotted line) and basically disappeared after replacement by the newly formed matrix. Abbreviations: BEP, bony endplate; CEP, cartilaginous endplate; NB, new bone; HE, haematoxylin and eosin; MT, Masson-trichrome; SF, Safranin O-Fast green; PSR, Picro-sirius red.

allografting although the findings in goats cannot be easily extrapolated to that in humans because of the difference in anatomy and biomechanics. We should acknowledge that healing and remodelling of the bony structure is not equivalent to the reestablishment of nutrient channels into the IVD allograft; the revascularisation and nutrient perfusion of the postoperative IVD allograft is still unclear which need further investigation. During transplantation, the growth plate in the recipient slot was removed while it was preserved in the IVD allograft. The growth plate could be found in the allograft after transplantation for 1.5 months and it disappeared completely after 6 months; nevertheless, it is still unclear whether the presence of growth plate will affect the healing of host-graft interface, the revascularisation as well as the nutrition reestablishment of the IVD allograft. The third limitation was that the cranial and caudal EPs were considered together; the difference between these two EPs was also controversial, especially in their thickness (Rodrigues et al., 2012). For IVD allograft transplantation, damage in whichever EP would result in loss of the integrity; hence, it should be reasonable to consider them together. Another limitation was that longitudinal morphological information (such as in-vivo magnetic resonance imaging (MRI) or in vivo CT scanning data) of the lumbar spine after IVD allografting was unavailable; these data will be meaningful to understand the loss of endplate integrity.

\section{Conclusions}

After intervertebral disc allograft transplantation, healing and remodelling of the bony structures ensured the stability and segment motion of the lumbar spine, but the integrity of bony and cartilaginous endplates was gradually lost and basically disappeared at the final follow-up.

\section{Acknowledgements}

We are very grateful to Mr. Jun-Ling Zeng from Nanfang Hospital, Guangzhou and Prof. Li Deng and Mrs. XiuQun Li from Sichuan University, Sichuan, P. R. China for kind support in this study. This work was financially supported by the Research Grants Council of Hong Kong (NO.: 773112) and the Tam Sai Kit Endowment Fund. Author contributions: conception and design: K.D.K.L. and Y.-C.H.; disc transplantation surgery: J.X., Y.-C.H., and K.D.K.L.; data collection: Y.-C.H. and J.X., V.Y. L., W.W.L., Y.H. and K.D.K.L.; data analysis and interpretation: Y.-C.H., K.D.K.L., J.X., V.Y. L., Y.H. and W.W.L.; writing, review and revision of the manuscript: Y.-C.H., K.D.K.L., V.Y. L., J.X., Y.H. and W.W.L. Financial support and final approval of the manuscript to be submitted: K.D.K.L. We wish to confirm that there are no known conflicts of interest associated with this publication and there has been no significant financial support for this work that could have influenced its outcome. 


\section{References}

Adams MA, Dolan P (2011) Biomechanics of vertebral compression fractures and clinical application. Arch Orthop Trauma Surg 131: 1703-1710.

Bauer TW, Muschler GF (2000) Bone graft materials. An overview of the basic science. Clin Orthop Relat Res 371: 10-27.

Boos N, Weissbach S, Rohrbach H, Weiler C, Spratt KF, Nerlich AG (2002) Classification of age-related changes in lumbar intervertebral discs. Spine 27: 2631-2644.

Delloye C, Cornu O, Druez V, Barbier O (2007) Bone allografts: What they can offer and what they cannot. J Bone Joint Surg Br 89: 574-579.

Di Bella C, Aldini NN, Lucarelli E, Dozza B, Frisoni T, Martini L, Fini M, Donati D (2010) Osteogenic protein-1 associated with mesenchymal stem cells promote bone allograft integration. Tissue Eng Part A 16: 2967-2976.

Ding Y, Ruan DK, He Q, Hou LS, Lin JN, Cui HP (2016) Imaging evaluation and relative significance in cases of cervical disc allografting: radiographic character following total disc transplantation. Clin Spine Surg: DOI: 10.1097/BSD.0b013e318290fc41.

Dolan P, Luo J, Pollintine P, Landham PR, Stefanakis M, Adams MA (2013) Intervertebral disc decompression following endplate damage: implications for disc degeneration depend on spinal level and age. Spine 38: 1473-1481.

Ferguson SJ, Steffen T (2003) Biomechanics of the aging spine. Eur Spine J 12 Suppl 2: S97-S103.

Fields AJ, Sahli F, Rodriguez AG, Lotz JC (2012) Seeing double: a comparison of microstructure, biomechanical function, and adjacent disc health between double- and single-layer vertebral endplates. Spine 37: E1310-1317.

Haschtmann D, Stoyanov JV, Gedet P, Ferguson SJ (2008) Vertebral endplate trauma induces disc cell apoptosis and promotes organ degeneration in vitro. Eur Spine J 17: 289-299.

Huang YC, Leung VY, Lu WW, Luk KD (2013) The effects of microenvironment in mesenchymal stem cellbased regeneration of intervertebral disc. Spine J 13: 352-362.

Huang YC, Urban JP, Luk KD (2014) Intervertebral disc regeneration: do nutrients lead the way? Nat Rev Rheumatol 10: 561-566.

Huang YC, Xiao J, Lu WW, Leung YL, Hu Y, Luk KD (2016) Lumbar intervertebral disc allograft transplantation: long-term mobility and impact on the adjacent segments. Eur Spine J: DOI: 10.1007/s00586-016-4535-z.

Junqueira LC, Montes GS, Sanchez EM (1982) The influence of tissue section thickness on the study of collagen by the Picrosirius-polarization method. Histochemistry 74: 153-156.

Kim JE, Shin JM, Oh SO, Yi WJ, Heo MS, Lee SS, Choi SC, Huh KH (2013) The three-dimensional microstructure of trabecular bone: Analysis of site-specific variation in the human jaw bone. Imaging Sci Dent 43: 227-233.

Lam SK, Xiao J, Ruan D, Ding Y, Lu WW, Luk KD (2012) The effect of remodeling on the kinematics of the malpositioned disc allograft transplantation. Spine 37: E357-366.

Lotz JC, Fields AJ, Liebenberg EC (2013) The role of the vertebral end plate in low back pain. Global Spine J 3: 153-164.

Luk KD, Ruan DK (2008) Intervertebral disc transplantation: a biological approach to motion preservation. Eur Spine J 17 Suppl 4: 504-510.

Messora M, Braga L, Oliveira G, Oliveira LF, Milagres R, Kawata L, Furlaneto F, Pola N, Campos N, Nagata M (2013) Healing of fresh frozen bone allograft with or without platelet-rich plasma: a histologic and histometric study in rats. Clin Implant Dent Relat Res 15: 438-447.

Miller JA, Schmatz C, Schultz AB (1988) Lumbar disc degeneration: correlation with age, sex, and spine level in 600 autopsy specimens. Spine 13: 173-178.

Moore RJ (2006) The vertebral endplate: disc degeneration, disc regeneration. Eur Spine J 15 Suppl 3: S333-337.

Odgaard A, Gundersen HJ (1993) Quantification of connectivity in cancellous bone, with special emphasis on 3-D reconstructions. Bone 14: 173-182.

Roberts S, Urban JP, Evans H, Eisenstein SM (1996) Transport properties of the human cartilage endplate in relation to its composition and calcification. Spine 21: 415-420.

Rodrigues SA, Wade KR, Thambyah A, Broom ND (2012) Micromechanics of annulus-end plate integration in the intervertebral disc. Spine J 12: 143-150.

Ruan D, He Q, Ding Y, Hou L, Li J, Luk KD (2007) Intervertebral disc transplantation in the treatment of degenerative spine disease: a preliminary study. Lancet 369: 993-999.

Rutges JP, Jagt van der OP, Oner FC, Verbout AJ, Castelein RJ, Kummer JA, Weinans H, Creemers LB, Dhert WJ (2011) Micro-CT quantification of subchondral endplate changes in intervertebral disc degeneration. Osteoarthritis Cartilage 19: 89-95.

Sakai D, Andersson GB (2015) Stem cell therapy for intervertebral disc regeneration: obstacles and solutions. Nat Rev Rheumatol 11: 243-256.

Urban JP, Smith S, Fairbank JC (2004) Nutrition of the intervertebral disc. Spine 29: 2700-2709.

Wade KR, Robertson PA, Broom ND (2012) On how nucleus-endplate integration is achieved at the fibrillar level in the ovine lumbar disc. J Anat 221: 39-46.

Wade KR, Robertson PA, Broom ND (2014) Influence of maturity on nucleus-endplate integration in the ovine lumbar spine. Eur Spine J 23: 732-744.

Wang Y, Battie MC, Boyd SK, Videman T (2011) The osseous endplates in lumbar vertebrae: thickness, bone mineral density and their associations with age and disk degeneration. Bone 48: 804-809.

Xiao J, Huang YC, Lam SK, Luk KD (2015) Surgical technique for lumbar intervertebral disc transplantation in a goat model. Eur Spine J 24: 1951-1958.

Yamaguchi T, Goto S, Nishigaki Y, Orias AA, Bae WC, Masuda K, Inoue N (2015) Microstructural analysis of three-dimensional canal network in the rabbit lumbar vertebral endplate. J Orthop Res 33: 270-276. 
Yoganandan N, Larson SJ, Gallagher M, Pintar FA, Reinartz J, Droese K (1994) Correlation of microtrauma in the lumbar spine with intraosseous pressures. Spine 19: 435-440.

\section{Discussion with Reviewers}

Reviewer I: The most striking result of this study was the loss of osseous endplate integrity at 6 and 12 months. The authors speculate that this could have been a result of normal bone remodelling or micro-fractures. However, in both cases, one would expect to also see quite a bit of new bone formation which would offset the loss of bone mass or bone integrity. With micro-fractures, micro-calluses should be evident. With remodelling, there should be new bone formation in the same regions as bone loss. Such a large amount of bone loss over 6 months is more likely a response due to a physical or biological environment unfavourable for bone. For example, could the non-viable allograft disc release factors causing bone resorption. Hence, did the authors look for further evidence of bone repair or turnover in the endplates and why do they think that this will eventually reverse or continue to degrade?

Authors: After cryopreservation, the metabolic activity was preserved in approximately $70 \%$ of the disc cells (Chan et al., 2010); in our previous primate study, viable cells in the postoperative disc allograft were recorded by histological results (Luk et al., 2003); in this goat study, cells with normal morphology in the subchondral bone, bony and cartilaginous endplates were also illustrated. Hence, the postoperative disc allograft is not a dead tissue-spacer to restore the spinal mobility only. But we still did not perform the study to investigate whether the disc allograft will release factors causing bone resorption in the bony endplate. In the Discussion section, we pointed out that bone remodelling or micro-fractures in the bony endplate may induce the loss of endplate integrity in the postoperative disc allograft. With remodelling, the first evidence was that new bone formation and bone loss in the bony endplate area were seen (Fig. 7G); nevertheless, the adjacent cartilaginous matrix did not provide an osteoinductive microenvironment for the local progenitor cells, leading to the imbalance of new bone formation and bone loss. This notion was supported by many studies in which the differentiation of progenitor cells in the discderived matrix was investigated; these scaffolds included extracellular nucleus matrix (Yuan et al., 2013), native (Liu et al., 2015) and decellularised nucleus matrix (Mercuri et al., 2013), and the whole decellularised disc organ (Chan et al., 2013). With micro-fractures, in this series of goat studies, we did not have evidence to show the formation of micro-calluses. Before the surgical technique of disc allografting was refined, micro-fracture in the bony endplate was found because of forceful insertion of allografts into the recipient slots. Hence, punching no matter how gentle is not acceptable in this surgery; however, at present we still do not have evidence to exclude that micro-fracture will not happen immediately after transplantation or before complete healing. We should acknowledge that loss of the endplate integrity should be multifactorial which needs further investigation. In our next research proposals, the factors related to bone remodelling and micro-fracture will be firstly considered based on our previous research experience; and in vivo $\mathrm{CT}$ scanning and ultrashort echo time (UTE)-MRI sequence will be utilised to observe the continuous changes of bony and cartilaginous endplates, respectively, after disc allografting in goats.

Reviewer I: In the discussion, the authors point out that the caprine vertebral endplate is different from that in humans. Unlike humans, goats have physes and epiphyses. Thus, the caprine allograft also contained these structures, which the human allograft would not have, and it is possible that revascularisation or lack thereof of such structures cause the loss in endplate integrity. However, this is not the only difference. In humans, the cartilaginous endplate is the growth plate and with skeletal maturation and closure of the apophyseal ring, this growth plate becomes quiescent. Hence, could the differences in the cartilaginous endplate between goats and humans lead to difficulties in translating these results to the human and why not or how?

Authors: Notably difference has been determined in the endplates across animal species and that of humans (Zhang et al., 2014). During transplantation, the growth plate in the recipient slot was removed while it was preserved in the IVD allograft; the growth plate could be found in the allograft after transplantation for 1.5 months and it disappeared completely after 6 months. We have pointed out this issue in the Discussion section; nevertheless, we have no idea whether the presence of growth plate causes the loss of endplate integrity in the postoperative IVD allograft. As mentioned in our previous paper (Huang et al., 2016), the goats are quadruped and do not reproduce the anatomy, loading, intradiscal pressures of the lumbar spine in the humans. Hence, we cannot easily extrapolate the results in this study to humans. The anatomical and functional difference in the cartilaginous endplate between goats and humans may be one of the major reasons; nevertheless, further investigation is required.

Reviewer II: How do the bony changes seen here - and the time scale - compare to those found in other bone allografts?

Authors: An IVD allograft usually carries 2-3 mm of vertebral bone at both the cranial and caudal ends in order to protect its integrity. Although the amount of bone transplanted during IVD allografting was very small, the continuous process of bone resorption and formation at the host-graft interface should result in the vertebral bone and the subchondral bone being similar with that of bone allografts. At 12-month follow-up, obvious differences in the Tb.N, Conn.Dn and DA were found when compared with the normal adjacent controls, suggesting constant remodelling at the bony structure to ensure the stability and mobility of the transplanted allograft. The dramatic change was the loss of endplate integrity, which has not been reported previously. 
Reviewer II: Can the authors suggest another mechanism, apart from micro-fractures, why the original architecture of the subchondral bone and bony endplate is destroyed during remodelling?

Authors: In the "Discussion" section, normal bony remodelling and micro-fractures were regarded as the major mechanisms for the loss of endplate integrity. The Reviewer was interested in the released factors from the allograft and their role in bone resorption of the bony endplate. We should acknowledge that loss of the endplate integrity should be multifactorial and at present we do not have solid evidences to unravel the underlying mechanisms of this phenomenon, especially the three mechanisms mentioned above. Further investigation is thus required.

Reviewer II: Would similar changes in the subchondral bone and endplate be expected in mature animals?

Authors: The life expectancy for goats is about 8 years, and goats at the age of 0.5-4 years are often used for preclinical studies in the area of disc degeneration and vertebroplasty. The goats are sexually mature as early as 6 months; and the 4-year goats still have the growth plates (Zhang et al., 2011), suggesting that the goat may still have growth capacity. Hence, we cannot easily define the skeletally mature in goats according to the age. It is still unclear about the natural history of intervertebral disc degeneration in goats; we thus do not know the natural change of subchondral bone and endplate with time and its effect on IVD allografting. The other difference between the young goats (used in this study) and mature ones comes from the presence of growth plates. During transplantation, the growth plate in the recipient slot was removed while it was preserved in the IVD allograft. The growth plate could be found in the allograft after transplantation for 1.5 months and it disappeared completely after 6 months; nevertheless, it is still unclear whether the presence of growth plate will affect the healing of the host-graft interface, the changes in the subchondral bone and endplate, the revascularisation, as well as the nutrition reestablishment of the IVD allograft.

\section{Additional References}

Chan SC, Lam SK, Leung VY, Chan D, Luk KD, Cheung KM (2010) Minimizing cryopreservation-induced loss of disc cell activity for storage of whole intervertebral discs. Eur Cell Mater 19: 273-283.

Chan LK, Leung VY, Tam V, Lu WW, Sze KY, Cheung KM (2013) Decellularized bovine intervertebral disc as a natural scaffold for xenogenic cell studies. Acta Biomater 9: 5262-5272.

Liu Y, Fu S, Rahaman MN, Mao JJ, Bal BS (2015) Native nucleus pulposus tissue matrix promotes notochordal differentiation of human induced pluripotent stem cells with potential for treating intervertebral disc degeneration. J Biomed Mater Res A 103: 1053-1059.

Luk KD, Ruan DK, Lu DS, Fei ZQ (2003) Fresh frozen intervertebral disc allografting in a bipedal animal model. Spine 28: 864-869.

Mercuri JJ, Patnaik S, Dion G, Gill SS, Liao J, Simionescu DT (2013) Regenerative potential of decellularized porcine nucleus pulposus hydrogel scaffolds: stem cell differentiation, matrix remodeling, and biocompatibility studies. Tissue Eng Part A 19: 952-966.

Yuan M, Yeung CW, Li YY, Diao H, Cheung KM, Chan D, Cheah K, Chan PB (2013) Effects of nucleus pulposus cell-derived acellular matrix on the differentiation of mesenchymal stem cells. Biomaterials 34: 3948-3961.

Zhang Y, Lenart BA, Lee JK, Chen D, Shi P, Ren J, Muehleman C, Chen D, An HS (2014) Histological features of endplates of the mammalian spine: from mice to men. Spine 39: E312-317.

Zhang Y, Drapeau S, An HS, Markova D, Lenart BA, Anderson DG (2011) Histological features of the degenerating intervertebral disc in a goat disc-injury model. Spine (Phila Pa 1976) 36: 1519-1527.

Editor's note: The Scientific Editor responsible for this paper was Mauro Alini. 